\title{
Simulation of Scoliosis Treatment Using a Brace
}

\section{J. Čulík}

Ivo Mařik M.D. has treated many child patients with scoliosis at the "Centre for Locomotor Defects", Olšanská 7, 130 00 Prague 3. The author has cooperated with him, and composed the computer program for the spine stress state under brace effects and for simulating scoliosis treatment. The program simulates the spinal curve remodelling in time for a specific child patients, and the algorithm for stress state calculation and treatment simulation is given.

Orthopaedists in the Czech Republic use Cheneau-type or Cerny-type corrective braces. The brace exerts force effects on the skeleton of a child. The brace is made individually for each patient, in the following way: first, a negative plaster form of the child's trunk is made, and then the positive plaster form is created. The orthopaedist determines the places where brace has to load the patient's trunk, and the plaster form deepened in these places on the basis of his advice. The laminate brace made according to this plaster form constricts the child's trunk (like a tight shoe).

This paper shows how the stress state is determined in vertebrae and in inter-vertebral discs, and the solution of spinal curvature correction under brace force effects for a specific child patient. The project aims to find the dependence of the activation and velocity of spinal curvature correction in the spinal stress state for many patients. The paper shows the computing algorithms for spinal deformations and the stress state under brace force effects, and a simulation of spinal curvature correction.

Spinal curvature is determined according to measured values on an X-ray of a patient before a brace is applied. The stress state in the spine and the spinal deformation are investigated by the finite element method as beam (spine) in an elastic ground (soft tissue). Two algorithms are used. The first algorithm deals with the spine above and below the soft tissues, and it is loaded by given displacements of the trunk surface. The second algorithm determines from the X-ray of a patient with and without a brace the spine deformation and the spine stress state, and the necessary trunk surface displacement is determined from this deformation.

The calculation algorithm and parameters were compared with contest of treatment. The trunk surface load was checked by sensor that plates were placed into the braces to measure the load values between the brace and the surface of the child. The simulation program assesses the spinal curvature correction according to the spinal curvature type, the spinal stress state and the period of time for which the brace will be applied.

Keywords: biomechanics, simulation of treatment, scoliosis, spine stress state, spine remodelling.

\section{Introduction}

Spinal corrective braces (see Fig. 1) are used for treating spine scoliosis in children (pathologic at deformation of chest curvature). The X-ray of the patient from Fig. 1 without and with a brace is shown in Fig. 2. Cheneau-type dynamic corrective braces or according to Cerny's patent No. 281800CZ (see Fig. 1) are usually used in the Czech Republic. The breast curvature can be classified according to King. A Chenau-type
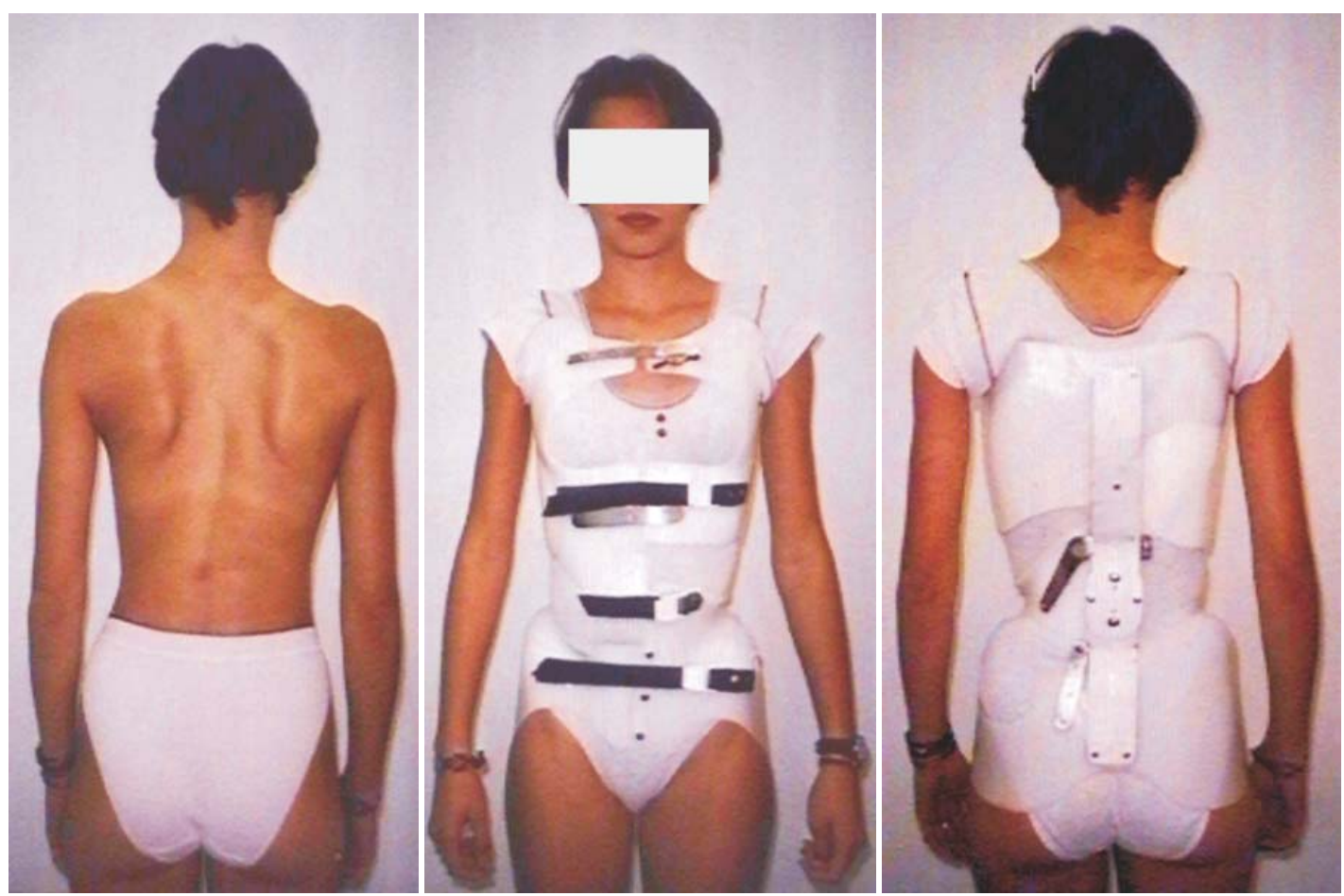

Fig. 1: Patient without and with the dynamic corrective brace according to Cerny's patent No. 281800CZ 
brace of is recommended for spinal curves of type King I, II, and IV types, a Cerny-type brace for spinal curves of King II, III and V types.

The brace constricts the child's trunk and makes a stress state in the patient's spine. The brace changes the spinal curvature, which means that the pathological spinal form is corrected. After long-term use of the brace, the spinal correction is permanent. The brace is made in the following manner: first, a plaster negative form and then a positive form of the child's trunk are made. According to his experience and the orthopedist's recommendation, the orthopedic assistant deepens the positive form of the plaster's in the place where the brace is to push on the child's trunk. The plastic brace is then made according to this plaster form. After it has been applicated to child's trunk, the brace constricts the places where the form has been deepened (the tight shoe principle).

If no computer search is used, the brace force effect is the result of the orthopaedist and his assistant's experience only, and it does not ensure that the form of the designed brace and the manner of treatment are optimal. This paper shows computer algorithms that are able to determine the stress state in vertebra and inter-vertebral discs, and spinal curve changes for specific brace applications. The theoretical conclusions are based on many causes of treatment. The remodelling of a pathological spine curvature depends on the spine stress state, and the time and manner in which the brace is applied. The course of treatment is simulated on the computer. The aim of this study is to determine the ideal brace form and a course of treatment course with the help of computer simulation. The computer program calculates the spine stress state and its curvature changes at each time point. The treatment simulation is now being provided simultaneously with the patient's treatment, and the computer model is being verified. If the computer model and the actual treatment have the same behaviour, then the model can be used for treatment prognosis in orthopaedic practice. Since the course of treatment takes a long time, the simulation model is still being verified so that its prognoses can become as precise as possible.

\section{Spinal curvature}

The task is solved using Cartesian coordinates $(x-$ spine axis direction, $y, z$ - frontal and saggital plane). The spinal curvature is stored in the computer as the following three functions

$$
y=y(x), \quad z=z(x), \quad \varphi=\varphi(x),
$$

where $\varphi$ is the turning based on the $\mathrm{x}$-axis. The spinal curvature can be described if the extreme values of $y$ and/or $z$ are measured from X-ray (the extremes of the white curvature in the X-ray on the left in Fig. 2). The method is also applied for the frontal and sagittal plane. The spinal curvature has 3 extremes of coordinate maxima. The curvature is divided into $n$ sectors between the beginning, the extremes and the end point of the coordinates, respectively (max. $n$ is 4 ). The ex-

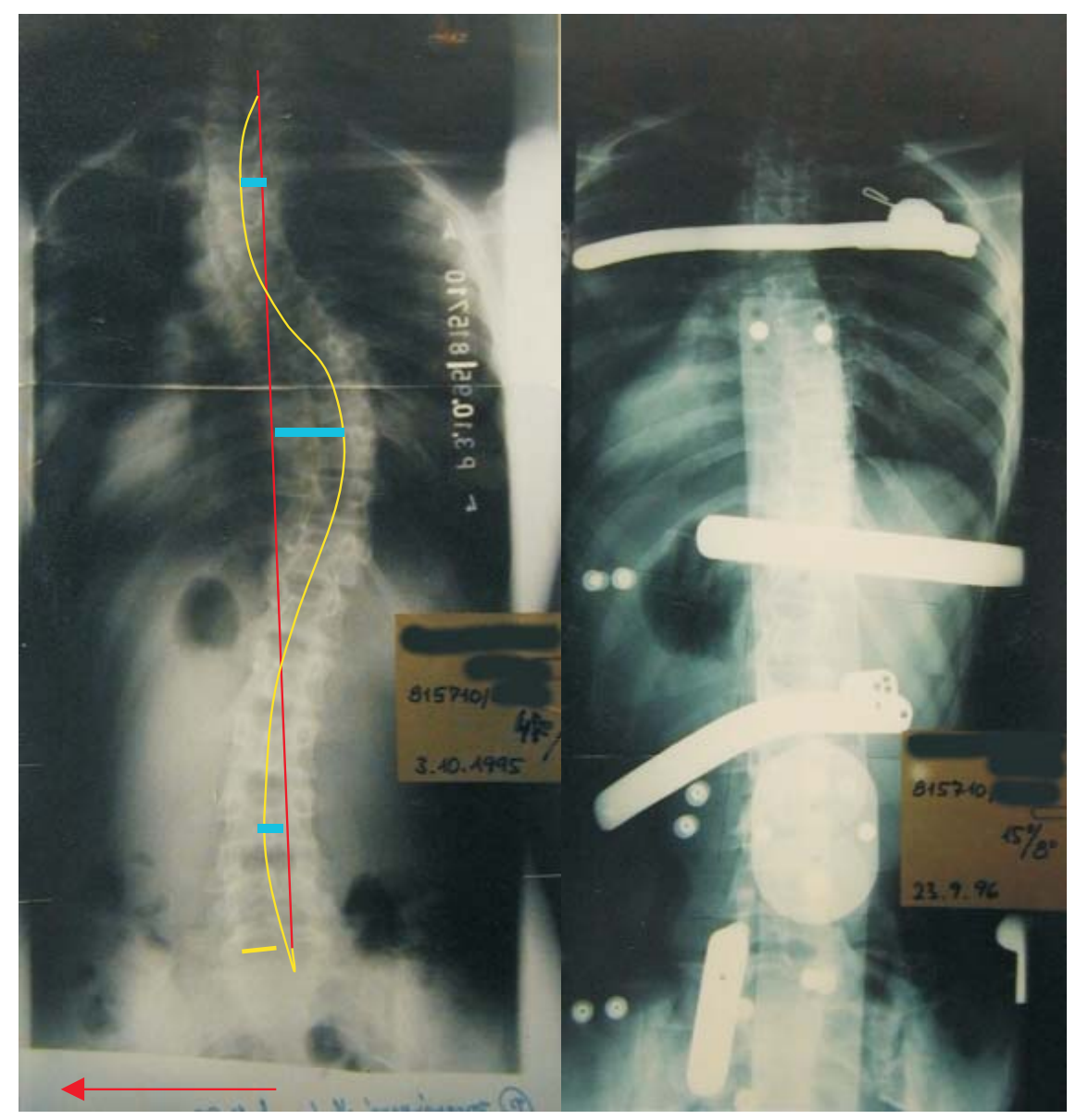

Fig. 2: Frontal X-ray of the patient from Fig. 1 without and with the corrective brace 
treme coordinates $x_{i}, y_{i}, i=1, \ldots, n-1$ and the coordinate $x_{n}$ of the spine end (spine length where $x_{n}=0$ ) are measured by $\mathrm{X}$-ray (see Fig. 2). The length of segment $i$ is

$$
l_{i}=x_{i}-x_{i-1} .
$$

The local coordinate $\xi$ is considered from the beginning of segment $i$. Function $y$ is considered as a polynomial. For the first segment (quadratic polynomial function) it is

$$
y=\frac{y_{i}}{l_{i}} \xi\left(2-\frac{\xi}{l_{i}}\right),
$$

for the inner segments (cubic polynomial function)

$$
y=y_{i-1} \frac{\left(y_{i}-y_{i-1}\right) \xi^{2}}{l_{i}^{2}}\left(3-2 \frac{\xi}{l_{i}}\right)
$$

and for the last segment (quadratic polynomial function)

$$
y=y_{i-1}\left(1-\frac{\xi^{2}}{l_{i}^{2}}\right) \text {. }
$$

\section{Deformation of the spine}

The inertia moment has to be determined for an inter-vertebral disc and a lignum cross-section area (see Fig. 3). The calculation procedure is as follows: the cross-section area is divided into triangles, and one third of triangular areas are concentrated to their side centres.

The spine is treated like a beam in an elastic basis, and the finite element method (deformation variant according to the Lagrange principle) is used for calculating the stress state. It is assumed that the vertebrae have no deformation. The potential energy is calculated for the inter-vertebral disc volume and for the compressed soft tissue region of a child's trunk. For simplification, the soft tissue is considered constant (rectangular cross-section of the trunk). The displacements and the turning at the vertebral centres are kinematic unknowns: $r=\left\{\begin{array}{l}r_{1} \\ r_{2}\end{array}\right\}, r_{1}^{T}=\left[\varphi_{x, i}, \varphi_{x, i+1}\right], r_{2}^{T}=\left[w_{i}, \varphi_{i}, w_{i+1}, \varphi_{i+1}\right]$,

where $\varphi_{\mathrm{x}}$ are turnings based on the spine axis. The following algorithm is valid for the frontal and sagittal planes, and planes will not be indicated by the plane index.

The stiffness matrix for the part of the spine between the centres of neighbouring vertebra is (torsion and beam influences)

$$
K=\left[\begin{array}{cc}
K^{1} & 0 \\
0 & K^{2}
\end{array}\right] .
$$

The sub-matrices will be determined separately for deformation of the spine and for soft tissue.

\section{Deformation of inter-vertebral discs}

The beam and torsion stiffness is

$$
k=\frac{2 E I}{l}, t=\frac{G I_{T}}{l},
$$

where $E, I$ are the modulus of elasticity and the moment of inertia of a cross-section of the inter-vertebral disc and lignums (see Fig. 3), and $l$ is the thickness of the disc. The influence of torsion is:

$$
K^{1}=\left[\begin{array}{cc}
t & -t \\
-t & t
\end{array}\right] .
$$

The influence for $\bar{R}$ - forces (moments) and $\bar{r}$ - movements (turns) on inter-vertebral disc boundaries are

$$
\bar{R}_{2}=\bar{K}^{2} \bar{r}_{2},
$$

The stiffness matrix $\bar{K}^{2}$ will be searched for inter-vertebral disc and kinematic value at the inter-vertebral borders. Because the vertebra are stiff, they have no potential energy. The inter-vertebral stiffness matrix will be recalculated on matrix $K^{2}$ for kinematic unknowns at the vertebra centres.

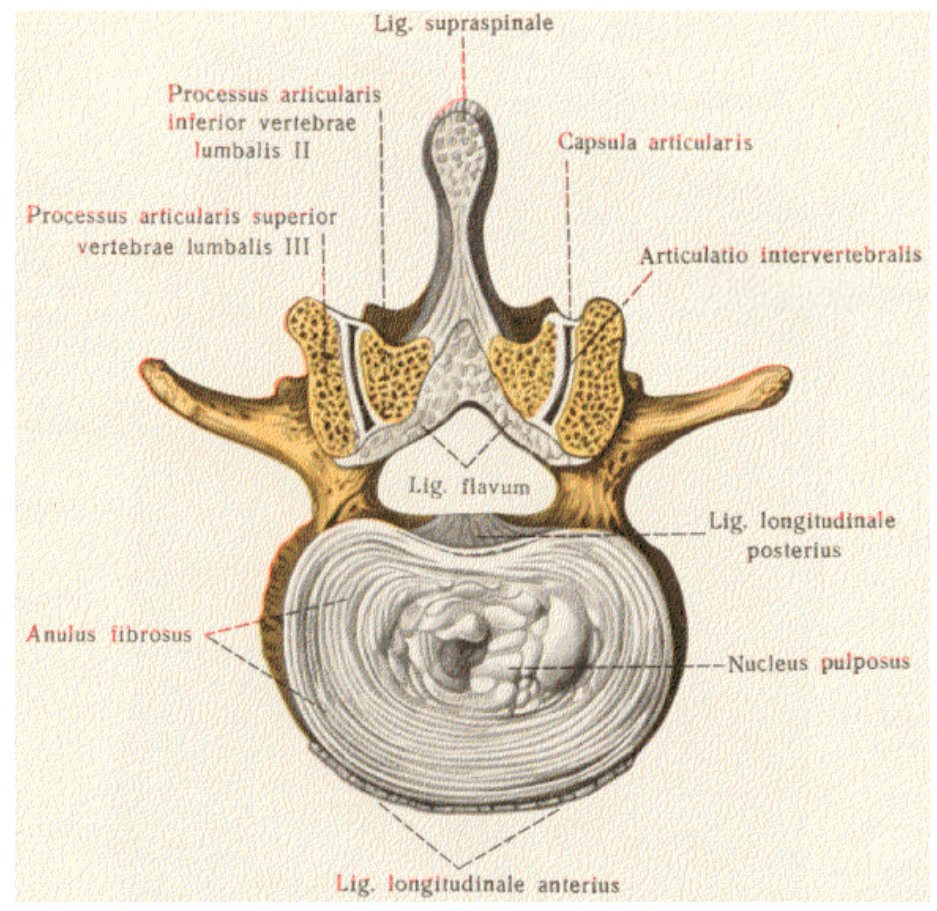

Fig. 3: Inter-vertebral disc and lignums 


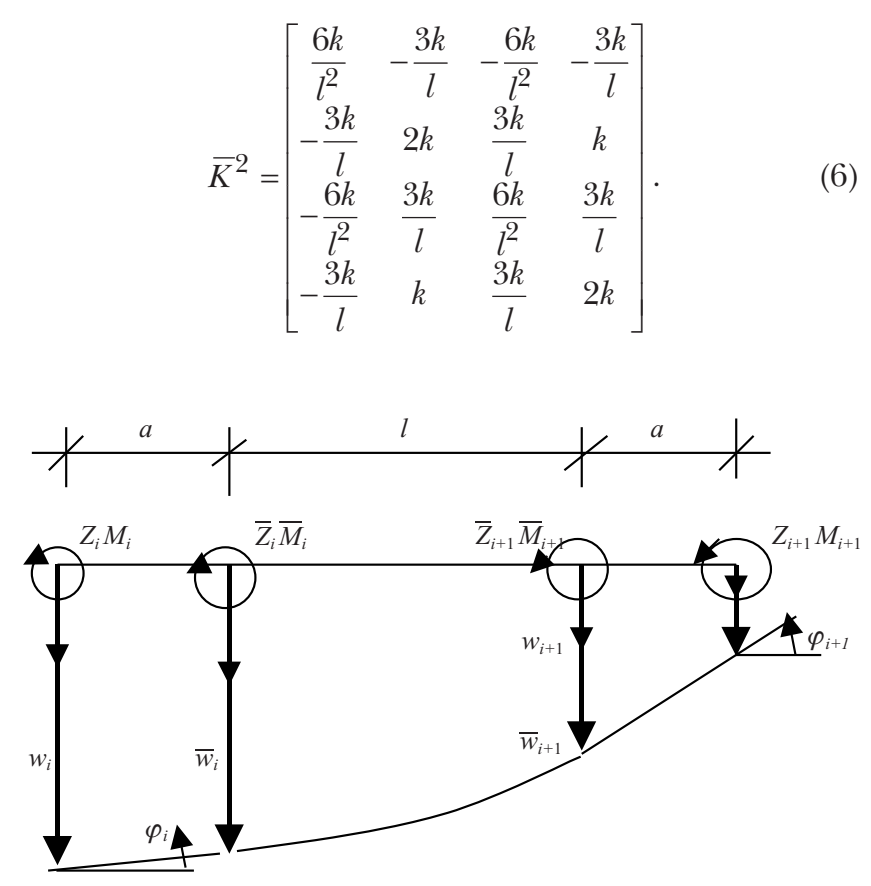

Fig. 4: Spine deformation is linear in the vertebra parts and curvilinear in the inter-vertebral part of the disc

Let the boundary forces $Z_{i}, M_{i}, Z_{i+1}, M_{i+1}$ and the kinematic unknowns $w_{i}, \varphi_{i}, w_{i+1}, \varphi_{i+1}$ be transformed from vertebra centres to values $\bar{Z}_{i}, \bar{M}_{i}, \bar{Z}_{i+1}, \bar{M}_{i+1}, \bar{w}_{i}, \bar{\varphi}_{i}, \bar{w}_{i+1}, \bar{\varphi}_{i+1}$ at the disc boundary points (see Fig. 4). As there is no deformation between the vertebral centre and the inter-vertebral disc boundary, the central spinal line is straight in this part, the spine movement $w$ has a linear course in the part of length $a$, and the torsion moment $M_{x}$ and turning $\varphi, \varphi_{\mathrm{x}}$ are invariable.

$\bar{w}_{i}=w_{i}-\bar{\varphi}_{i} a, \bar{w}_{i+1}=w_{i+1}+\bar{\varphi}_{i+1} a, \bar{\varphi}_{i}=\varphi_{i}, \bar{\varphi}_{i+1}=\varphi_{i+1}$,

$\bar{M}_{i}=M_{i}+Z_{i} a, \bar{M}_{i+1}=M_{i+1}+Z_{i+1} a, \bar{Z}_{i}=Z_{i}, \bar{Z}_{i+1}=Z_{i+1}$.

Let us put (7), (8) to (5)

$$
R_{2}+\left\{\begin{array}{c}
0 \\
a Z_{i} \\
0 \\
-a Z_{i+1}
\end{array}\right\}=\bar{K}^{2}\left(r_{2}\left\{\begin{array}{c}
-a \varphi_{i} \\
0 \\
a \varphi_{i+1} \\
0
\end{array}\right\}\right) .
$$

Formula (9) can be written

$$
R_{2}=K^{2} r_{2}
$$

where $K^{2}$ is the stiffness matrix for vertebra centres

$$
K^{2}=\left[\begin{array}{cccc}
\frac{6 k}{l^{2}} & -\frac{3 k}{l}\left(\frac{2 a}{l}+1\right) & -\frac{6 k}{l^{2}} & -\frac{3 k}{l}\left(\frac{2 a}{l}+1\right) \\
-\frac{3 k}{l}\left(\frac{2 a}{l}+1\right) & k\left[2+\frac{3 a}{l}\left(\frac{2 a}{l}+1\right)\right] & \frac{3 k}{l}\left(\frac{2 a}{l}+1\right) & k\left[2+\frac{3 a}{l}\left(\frac{2 a}{l}+1\right)\right] \\
-\frac{6 k}{l^{2}} & \frac{3 k}{l}\left(\frac{2 a}{l}+1\right) & \frac{6 k}{l^{2}} & \frac{3 k}{l}\left(\frac{2 a}{l}+1\right) \\
-\frac{3 k}{l}\left(\frac{2 a}{l}+1\right) & k\left[2+\frac{3 a}{l}\left(\frac{2 a}{l}+1\right)\right] & \frac{3 k}{l}\left(\frac{2 a}{l}+1\right) & k\left[2+\frac{3 a}{l}\left(\frac{2 a}{l}+1\right)\right]
\end{array}\right] .
$$

Analogous formulas are valid for the $y$ axis direction.

\subsection{Compressed Soft Tissue (Elastic Ground)}

The compressed soft tissue of a child's trunk round the spine is considered as an elastic ground according to [1] p. 86-113, and the final formulas will be used here. The width of the ground is considered constant. Let us calculate the parameters

$$
\begin{gathered}
C_{1}=\frac{E_{P}}{h}, \quad C_{2}=\frac{E_{P} h}{6}, \\
C_{1}^{*}=C_{1}+\frac{1}{b} \sqrt{C_{1} C_{2}}, \quad C_{2}^{*}=C_{2}+\frac{1}{2 b} \sqrt{\frac{C_{2}^{3}}{C_{1}}}, \\
C_{3}^{*}=\frac{1}{3} C_{1} b^{2}+C_{2}+b \sqrt{C_{1} C_{2}}, \quad C_{4}^{*}=\frac{1}{3} C_{2} b^{2}+\frac{b}{2} \sqrt{\frac{C_{2}^{3}}{C_{1}}},
\end{gathered}
$$

where $E_{P}, h, b$ are the modulus of elasticity, thickness and width of the compressed soft tissue. The torsion stiffness sub-matrix is:

$$
K^{1}=\frac{b l}{3} C_{3}^{*}\left[\begin{array}{ll}
2 & 1 \\
1 & 2
\end{array}\right]+\frac{2 b}{l} C_{4}^{*}\left[\begin{array}{rr}
1 & -1 \\
-1 & 1
\end{array}\right] .
$$

The beam stiffness sub-matrix is:

$$
\begin{aligned}
K^{2}=2 b l C_{1}^{*}\left[\begin{array}{cccc}
\frac{13}{25} & -\frac{11 l}{210} & \frac{9}{70} & \frac{13 l}{420} \\
-\frac{11 l}{210} & \frac{l^{2}}{105} & -\frac{13 l}{420} & -\frac{l^{2}}{140} \\
\frac{9}{70} & -\frac{13 l}{420} & \frac{13}{35} & \frac{11 l}{210} \\
\frac{13 l}{420} & -\frac{l^{2}}{140} & \frac{11 l}{210} & \frac{l^{2}}{105}
\end{array}\right]+ \\
+\frac{2 b C_{2}^{*}}{l}\left[\begin{array}{cccc}
\frac{6}{5} & -\frac{l}{10} & -\frac{6}{5} & -\frac{l}{10} \\
-\frac{l}{10} & \frac{2 l^{2}}{15} & \frac{l}{10} & \frac{l^{2}}{30} \\
-\frac{6}{5} & \frac{l}{10} & \frac{6}{5} & \frac{l}{10} \\
-\frac{l}{10} & -\frac{l^{2}}{30} & \frac{l}{10} & \frac{2 l^{2}}{15}
\end{array}\right]
\end{aligned}
$$

\section{The first algorithm}

The brace constricts the trunk in the place where the plaster positive form of the child's trunk has been deepened; this means that the trunk surface (soft tissue surface) has non-zero prescribed displacements in these places. Let us assume that the prescribed displacement acts from above for a lying patient, and the $z$-axis direction is from below. The compression of the soft tissue up the spine is $w_{0}-w$ and below it is $w$, where $w$ is the spine displacement and $w_{0}$ is the prescribed trunk surface displacement. Let matrices $K_{\text {above }}, K_{\text {below }}$ be calculated according to formulas (12), (13) for the part of the trunk above and below the spine. The potential energy of the soft tissue part is

$$
\begin{aligned}
\delta E_{P} & =\delta r^{T}\left[-K_{\text {above }}\left(r_{0}-r\right)+K_{\text {below }} r\right]= \\
& =\delta r^{T}\left[-K_{\text {above }} r_{0}+\left(K_{\text {below }}+K_{\text {above }}\right) r\right] .
\end{aligned}
$$

The term $K_{\text {above }} r_{0}$ can be calculated, and its negative form can be considered as a load vector (the right side of linear algebraic equations of the finite element method). In this way, the potential energy can be considered in the compressed parts of the soft tissue only; this means that the terms $K_{\text {above }}\left(r_{0}-r\right)$ and/or $K_{\text {below }} r$ are taken into cosideration only if they are positive. An iteration calculation is necessary for correct results; this means that the load vector is calculated for 
the compressed soft tissue part above and/or below the spine according to the results from the last iteration step.

The oblique load will be searched. Let $y, z$ be coordinates of the point of the center where the positive plaster form was deepened, and let $\Delta$ be the depth to which the plaster positive form has been deepened in the perpendicular direction to the child's trunk surface. Now, $\Delta$ is a prescribed trunk surface displacement and $y, z$ are its coordinates (positive displacement is in the direction from the trunk surface to the spine). Let us consider that the transversal cross-section of the trunk has a half elliptic form with radiuses $a, b$ for $z>0$ and $a, \bar{b}$ for $z<0$. The following formulas can be written for the ellipse

$$
z=b \sqrt{1-\frac{y^{2}}{a^{2}}}
$$

If formula (13) is derived, angle $\varphi$ of the tangent with axis $y$ can be calculated; the negative value of angle $\varphi$ is the angle of the normal with the $z$ axis.

$$
\operatorname{tg} \varphi=z^{\prime}=-\frac{b y}{a \sqrt{a^{2}-y^{2}}} .
$$

The prescribed surface displacements $v_{0}, w_{0}$ in $y, z$ directions are

$$
v_{0}=-\Delta \sin \varphi, \quad w_{0}=\Delta \cos \varphi .
$$

The problem can be solved in the $x, y$ or $x, z$ plane with prescribed displacements $v_{0}$, or $w_{0}$ or more correctly as a space problem with a space spine and soft tissue elements. The stiffness matrix for the space spine element can be considered in the same way as formulas (4), (5) and (11), but the matrix $K^{2}$ (see (11)) also has the elements for the direction of the axis $y$. As the vertebra have no deformations, the kinematic variables at the vertebra surface can be calculated from the kinematic variables of the centre of gravity of the vertebra (see (3)). The normal and tangential stresses on the boundary between a vertebral and an inter-vertebral disc are then calcu- lated from the resulting joint forces and moments. The normal force of the $x$-axis load has to be taken into account in the normal stress calculation, too, and the influence of shear and torsion should be taken into account in the tangent stress calculation.

The parameters and calculation algorithm are being verified with values observed in the X-ray of a child with and without a brace, i.e., the calculated function values $y, z$ and $y+v$, $z+w$ and their extremes are compared with the patient's $\mathrm{X}$-ray.

\section{The second algorithm}

The spinal curvature extremes are measured on X-rays taken without and with a brace. The spinal curvature coordinates are determined for the X-rays, and the spine deformation function $r_{2}$ is the difference of the two curvature coordinates. The joint force (moment) vector $R_{2}$ can be calculated from (10) and beam theory can be used to calculate the spine stress state. If displacement vector $w$ is known, we can calculate from (14) vector $w_{0}$ - the necessary displacement of the trunk surface and the most suitable place for deepening the brace.

\section{Simulation of treatment}

If the brace is removed from the child's trunk after being applied for some time, then the spine does not return to its previous position, but the pathological spine form is partly corrected. An example of the result of scoliosis treatment of a King I type spinal defect is shown in Fig. 5. The maximum of the angles between the spine axis has been measured at the thoracic and lumbar parts of spine. The measured angles were 27 grads and 34 grads before treatment; 2 and 5 grads with the brace; 9 and 12 grads after treatment. Statistical data for various spinal curve types according to King-Moe are given in Table 1.

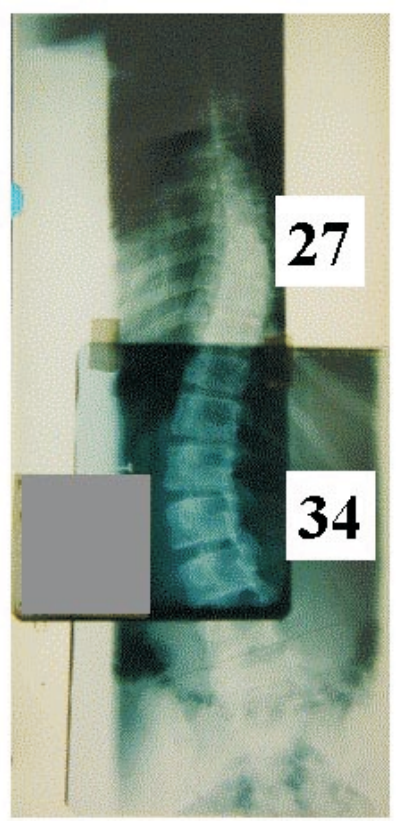

14.5 years

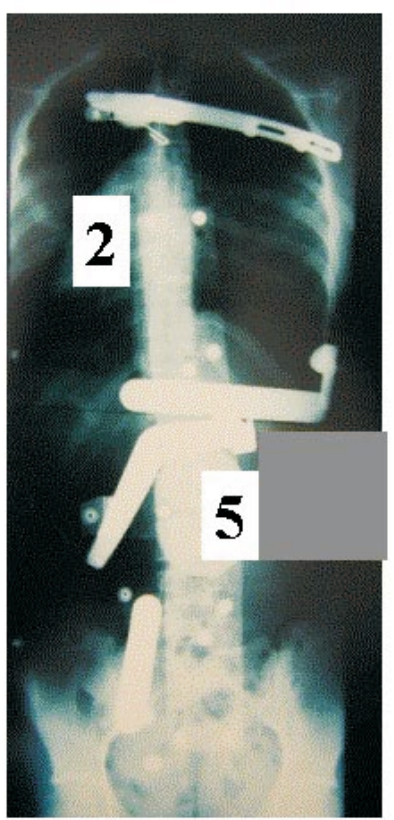

15 years

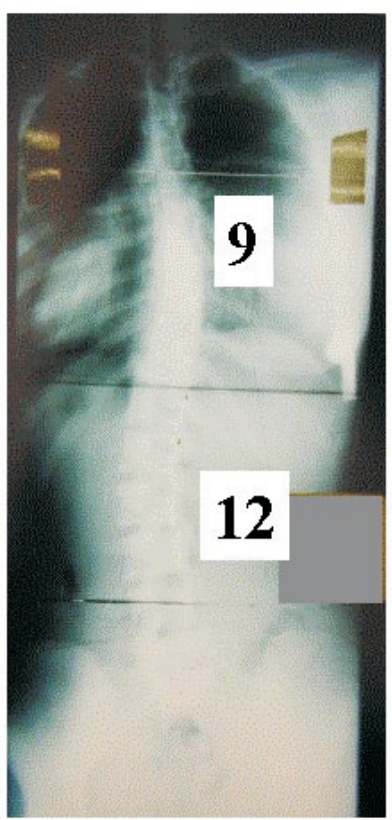

17 years

Fig. 5: X-rays of a patient with King I type spinal curvature, before treatment, with a brace, and after 2.5 years 
Table 1: Statistical data for spinal angle correction after scoliosis treatment with braces

\begin{tabular}{|c|l|l||}
\hline King - Moe & \multicolumn{1}{|c|}{ Th \% (No.) } & $\mathrm{L} \%$ (No.) \\
\hline I & $52_{(87)} / \mathbf{5 9}_{(47)}$ & $\underline{60_{(87)} / 5_{(47)}}$ \\
\hline II & $3_{(32)} / 35_{(19)}$ & $53_{(32)} / 5_{(19)}$ \\
\hline III & $48_{(46)} / 48_{(58)}$ & $47_{(46)} / 49_{(58)}$ \\
\hline IV & $\underline{62}(10) / 47_{(5)}$ & $47_{(10)} / 47_{(5)}$ \\
\hline V & $11_{(2)} / 3_{(2)}$ & $13_{(2)} / \underline{60}_{(2)}$ \\
\hline CHÊNEAU/CERNY & $49_{(177)} / 49_{(131)}$ & $\underline{56}(177) / 49_{(13)}$ \\
\hline
\end{tabular}

The second column is for the thoracic part of the spine, and the third column is for the lumbar part of the spine. The first set of data is for $\mathrm{Ch}$ neau and the second set of data for Cerny brace types. The percentages of angle correction are given first, followed by the number of patients treated, in parenthesses. The prognosis prognosis and speed of the treatment effect are made according to the statistical data and the spine stress state. The condition for succesful treatment is periodical use of the brace in accordance with orthopaedic advice.

\section{Conclusion}

Many child patients have been observed within this project, and the dependence between spinal curvature correction, the spine stress state and the time interval of applying the brace have been studied. Theoretical conclusions about spine remodelling have been sought. The computer simulation model and its parameters are being verified to ensure that the behaviour of the model is the same as the child's course of treatment. Since the treatment takes a long time, the theoretical conclusions can only be determined after a sufficient number of comparison have been made between observed treatment courses and their computer simulations.

\section{Acknowledgment}

This research was supported by grant MSM 21-0000012 "Trans-disciplinary Research in Biomedical Engineering, Area" and is being conducted in cooperation with ortho- peadist and pediatrician Ivo Mařík, M.D., PhD. (Center for Defects of the Locomotor Apparatus, Olšanská 7, 13000 Prague 3), who has treated the patients, and Ing. Pavel Černý (Orthotic Center, Truhlářská 8, 11000 Prague 1), who has made the special braces and measurement instruments.

\section{References}

[1] Bittnar Z., Šejnoha J.: Numerical Method in Mechanics (in Czech). Prague: 1.Ed. CTU, 1992.

[2] Černý P., Mařík I., Zubina P., Hadraba I.: “Application of Orthotic as a Technical Device of Rehabilitation by Bone Displasies." (in Czech) Locomotor Systems, Vol. 5 (3-4), Prague 1998, p.145-151.

[3] Čulík J.: "Computer Simulation of Spine Deformities Treatment with Orthoses". Word Congress on Computer Mechanics, "WCCM - Books of Abstracts" Vol. I., International Association for Computer Mechanics, Vienna University of Technology, p. 303, full paper at http://wccm.tuwien.ac.at., Vienna 2002.

[4] Čulík J., Mařík I., Černý P., Zubina P., Zemková D.: "Computer Control of Bone Deformities Treatment by Limb Orthoses". Journal of Musculoskeletal \& Neuronal Interaction. Vol. 2 No. 4, Nafplion 2002, p. 389.

[5] Denis, F.: "Spinal Instability as Acute Spinal Trauma." Clin. Orthop., Vol. 189, 1984, p. 65.

[6] Ch neau, J.: "Bracing Scoliosis". Locomotor Systems, Vol. 5, No. 1-2, Prague 1998, p. 60-73.

[7] Mařík I., Černý P., Sobotka Z., Korbelař P., Kuklík M., Zubina P.: "Conservative Therapy of Spine Deformities with Dynamic Trunk Orthoses." (in Czech). Locomotor Systems. Vol. 3, No. 1, Prague 1996, p. 38-41.

[8] Roy-Camille R.: Rachis dorsolumbale traumatigue non neurologique. Paris: Masson, 1980.

[7] Zubina P.: "Prevention of Spine Deformities after Multiply Level Laminecktonii at Child Age”. Locomotor Systems, Vol. 4, No. 2, Prague 1997, p. 3-18.

Prof. Ing. Jan Čulík, DrSc.

phone: +420224354 481, +420312608208

e-mail:culik@ubmi.cvut.cz

Czech Technical University in Prague

Institute of Biomedical Engineering

náměstí Sítná 3105 / 610

27201 Kladno 2, Czech Republic 